\title{
Evolution of open charm production with event multiplicity with ALICE
}

\author{
Marco Giacalone ${ }^{a, b, *}$ for the ALICE Collaboration \\ ${ }^{a}$ Istituto Nazionale di Fisica Nucleare (INFN), \\ Viale Berti Pichat 6/2, Bologna, Italy \\ ${ }^{b}$ Department of Physics, University of Bologna, \\ Via Irnerio 46, Bologna, Italy \\ E-mail: mgiacalo@cern.ch
}

The study of charm production as a function of multiplicity is important to investigate the role of multi-parton interactions, color-reconnection mechanisms, and spectra modification in high multiplicity events with respect to minimum bias ones, and to dig into the possible presence of collective phenomena in high-multiplicity environments.

Moreover, charm cross-section measurements in pp collisions provide a useful test of QCD calculations while measurements in $\mathrm{p}-\mathrm{Pb}$ collisions are useful to analyze Cold Nuclear Matter effects.

In this contribution, recent measurements of the evolution of the production yields with the event charged-particle multiplicity of prompt D-mesons and leptons from heavy-flavour hadron decays in pp collisions at $\sqrt{s}=13 \mathrm{TeV}$ are discussed. The results are compared to the $\mathrm{J} / \Psi$ measurement and to model calculations. The $\Lambda_{c}^{+} / \mathrm{D}^{0}$ and $\mathrm{D}_{\mathrm{s}} / \mathrm{D}^{0}$ yield ratios as a function of multiplicity in pp collisions at $\sqrt{s}=13 \mathrm{TeV}$ are also presented. The measurements in $\mathrm{p}-\mathrm{Pb}$ of the centralitydependence of the D-meson nuclear modification factor and of the elliptic flow of heavy-flavour decay leptons in high-multiplicity events are also discussed.

\footnotetext{
*** 10th International Workshop on Charm Physics (CHARM2020), ***

*** 31 May - 4 June, 2021 ***

*** Mexico City, Mexico - Online ***
}

\footnotetext{
${ }^{*}$ Speaker
} 


\section{Introduction}

Measurements of charm meson and baryon production in proton-proton collisions are an important test for perturbative QCD calculations. They provide also the reference for measurements in $\mathrm{p}-\mathrm{Pb}$, a collision system important to investigate cold nuclear matter effects (like nuclear modification of parton distribution functions). Furthermore, the study of the charm production in pp and $\mathrm{p}-\mathrm{Pb}$ collisions as a function of the charged-particle multiplicity of the event allows the investigation of effects related to multiple parton interactions and of color reconnection, which are expected to play a relevant role in charged particle production at high energy at the LHC. Possible modifications of the spectra in high-multiplicity $\mathrm{pp}$ and $\mathrm{p}-\mathrm{Pb}$ collisions with respect to minimum bias ones might signal the presence of radial flow and collective phenomena typically observed in $\mathrm{Pb}-\mathrm{Pb}$ collisions. The excellent particle identification and tracking capabilities of A Large Ion Collider Experiment (ALICE) [1] are exploited to analyse several charm-hadron decay channels, namely:

- hadronic decays: $\mathrm{D}^{0} \rightarrow \mathrm{K}^{-} \pi^{+} ; \mathrm{D}^{+} \rightarrow \mathrm{K}^{-} \pi^{+} \pi^{-} ; \mathrm{D}^{*+} \rightarrow \mathrm{D}^{0} \pi^{+} \rightarrow \mathrm{K}^{-} \pi^{+} \pi^{-} ; \mathrm{D}_{\mathrm{s}}^{+} \rightarrow \phi \pi^{+} \rightarrow$ $\mathrm{K}^{-} \mathrm{K}^{+} \pi^{+} ; \Lambda_{c}^{+} \rightarrow \mathrm{pK}^{-} \pi^{+} ; \Lambda_{c}^{+} \rightarrow \mathrm{pK}_{\mathrm{s}}^{0} ; \Sigma_{c}^{0} \rightarrow \Lambda_{c}^{+} \pi^{-} ; \Sigma_{c}^{++} \rightarrow \Lambda_{c}^{+} \pi^{+}, \Xi_{c}^{0} \rightarrow \Xi^{-} \pi^{+} ;$ $\Xi_{c}^{+} \rightarrow \Xi^{-} \pi^{+} \pi^{+} ; \Omega_{c}^{0} \rightarrow \Omega^{-} \pi^{+}$.

- semileptonic decays: B and D mesons $\rightarrow \mathrm{e}+\mathrm{X}$; B and D mesons $\rightarrow \mu+\mathrm{X} ; \Xi_{c}^{0} \rightarrow \Xi^{-} \mathrm{e}^{+} v_{\mathrm{e}}$; $\Lambda_{c}^{+} \rightarrow \Lambda \mathrm{e}^{+} v_{\mathrm{e}}$

The particle identification in the central barrel (pseudorapidity $|\eta|<0.9$ ) is provided by the Time Projection Chamber (TPC) and the Time Of Flight (TOF) detectors, as well as by the Electromagnetic Calorimeter (EMCal) for high-pt electron identification. The track position is determined with high resolution by the Inner Tracking System (ITS) and TPC. At forward rapidity $(2.5<y<4)$, muons are identified and reconstructed in the muon spectrometer.

The Silicon Pixel Detector (SPD) and the V0 detectors are used to estimate the multiplicity, which is defined as:

- SPD tracklets: track segments reconstructed from two hits in the detector layers (used at $|\eta|<1)$

- V0M percentile: multiplicity based on the percentile distribution of the V0 amplitude, which is the weighted sum of charge deposition in the V0A and V0C scintillators $(2.8<\eta<5.1$ and $-3.7<\eta<-1.7)$.

The multiplicity intervals estimated with both methods are converted to mean charged particle multiplicity density $\left(\left\langle\mathrm{dN}_{\mathrm{ch}} / \mathrm{d} \eta\right\rangle\right)$ of charged-primary particles $\mathrm{N}_{\mathrm{ch}}$.

\section{Charm production in pp collisions}

In this section, the charm production measurements as a function of multiplicity are discussed. Results will be expressed using the so-called self-normalised yield, defined as

$$
\frac{\mathrm{d}^{2} N_{\text {mult }} /\left(\mathrm{d} y \mathrm{~d} p_{\mathrm{T}}\right)}{\left\langle\mathrm{d}^{2} N_{\text {int }} /\left(\mathrm{d} y \mathrm{~d} p_{\mathrm{T}}\right)\right\rangle}=\frac{Y_{\text {mult }}}{Y_{\text {int }}}
$$


where $Y_{\text {mult/int }}$ is the corrected yield respectively in multiplicity intervals and in the multiplicityintegrated case.

The measurements of D-meson and non-prompt $\mathrm{J} / \psi$ production as a function of multiplicity in $\mathrm{pp}$ collisions at $\sqrt{s}=7 \mathrm{TeV}$ were reported in [2]: the self-normalised yields of both particle species show a faster than linear increasing trend. Within uncertainties, the self-normalised yield evolution with multiplicity does not depend significantly on $p_{\mathrm{T}}$.

The results for D mesons at $\sqrt{s}=13 \mathrm{TeV}$ are shown in Fig. 1 in six $p_{\mathrm{T}}$ intervals up to $24 \mathrm{GeV} / c$. A dependence on $p_{\mathrm{T}}$ is observed (thanks to the better precision of $13 \mathrm{TeV}$ results compared to $7 \mathrm{TeV}$ ones), and high- $p_{\mathrm{T}}$ intervals show a stronger increase of the self-normalised yields with multiplicity.

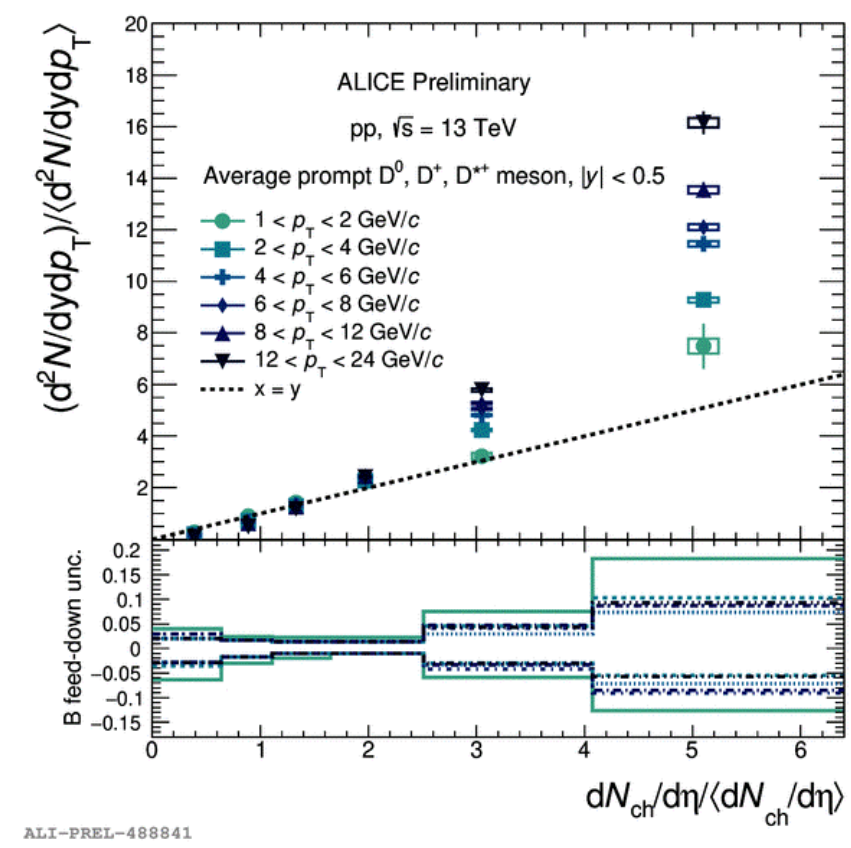

Figure 1: Average of $\mathrm{D}^{0}, \mathrm{D}^{+}, \mathrm{D}^{*+}$ meson self-normalised yields vs. charged particle multiplicity in pp collisions at $\sqrt{s}=13 \mathrm{TeV}$ at midrapidity in different $p_{\mathrm{T}}$ intervals. The b-hadron feed-down systematic uncertainties are shown in the bottom panel.

The average of $\mathrm{D}^{0}, \mathrm{D}^{+}, \mathrm{D}^{*+}$ meson results are compared to different Monte Carlo models in Fig. 2. The EPOS3 generator [3] with flux-tube initial conditions, generated in the Gribov-Regge multiple scattering framework, and hydrodynamical evolution describes reasonably well data, while EPOS3 without hydro and the 3-pomeron Color Glass Condensate (CGC) [4] respectively underestimates and overestimates them.

The self-normalised yields as a function of multiplicity of heavy-flavour hadron decay electrons in pp collisions at midrapidity at $13 \mathrm{TeV}$ measured for $p_{\mathrm{T}}<4.5 \mathrm{GeV} / c$ are reported in Fig. 3 and compared to the default PYTHIA 8.2 tune, Monash 2013, in which both color reconnection (CR) and multiple parton interactions are included.

Furthermore, self-normalised yields of heavy-flavour hadron decay muons at forward rapidity $(2.5<y<4)$ vs. multiplicity at $\sqrt{s}=8 \mathrm{TeV}$ in 5 different $p_{\mathrm{T}}$ intervals are reported in Fig. 4 compared to EPOS prediction without the hydrodynamical evolution enabled. 


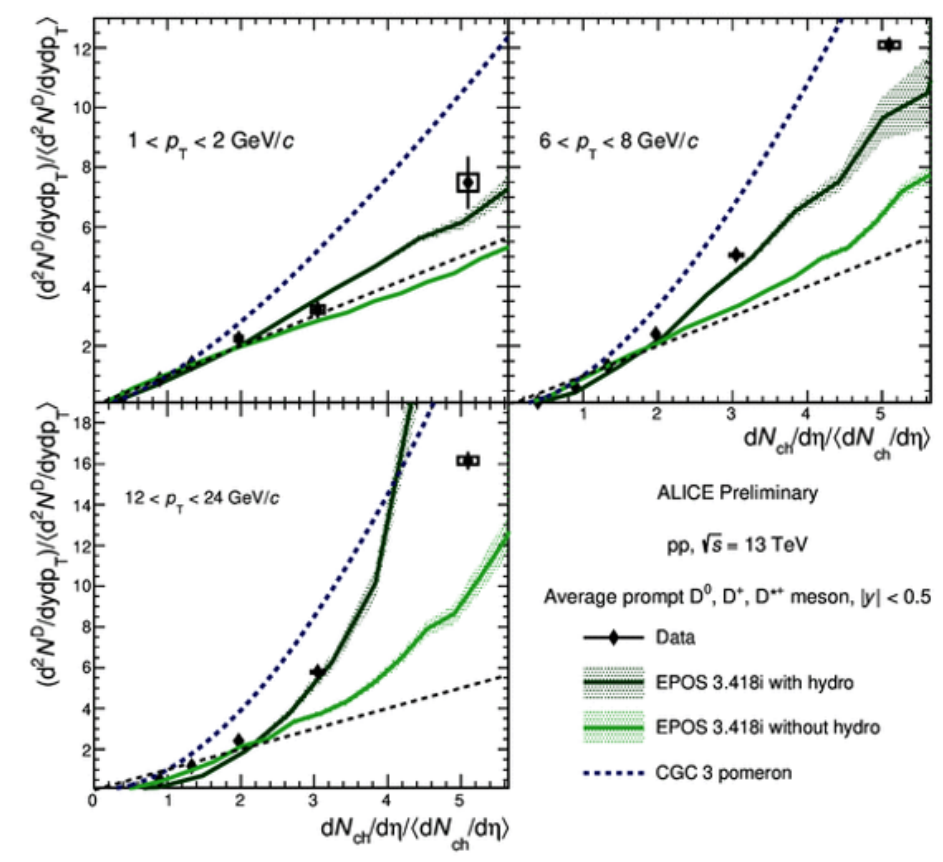

ALI-PREL-488879

Figure 2: Comparison of D-meson self-normalised yields as a function of charged-particle multiplicity to models data in three $p_{\mathrm{T}}$ intervals in pp collisions at $\sqrt{s}=13 \mathrm{TeV}$.

Heavy-flavour electrons are well described by PYTHIA 8.2 data, while muons are underestimated by EPOS. Both measurements show a faster than linear trend vs. multiplicity. In both cases the multiplicity refers to the charged particles one in $|\eta|<1$.

The self-normalised yields of $\mathrm{J} / \psi$, D-meson average and heavy-flavour decay electrons compared in similar $p_{\mathrm{T}}$ intervals in Fig. 5 show similar trends.

In this contribution the production ratios $\Lambda_{\mathrm{c}}^{+} / \mathrm{D}^{0}$ and $\mathrm{D}_{\mathrm{s}}^{+} / \mathrm{D}^{0}$ (Fig. 6) in pp collisions at $13 \mathrm{TeV}$ are also presented. The results show a multiplicity dependence of the $\Lambda_{\mathrm{c}}^{+} / \mathrm{D}^{0}$ yield ratio that hints to a modification of hadronization mechanisms with multiplicity. The PYTHIA 8.2 Monash tune strongly underestimates the data, and does not reproduce the trend with $p_{\mathrm{T}}$, while the CR mode 2 tune [5] qualitatively describes the $p_{\mathrm{T}}$ shape and multiplicity trend.

On the other hand, the $\mathrm{D}_{\mathrm{s}}^{+} / \mathrm{D}^{0}$ ratio shows no significant multiplicity dependence.

\section{Measurements in $\mathbf{p}-\mathbf{P b}$ collisions}

An important observable in $\mathrm{p}-\mathrm{Pb}$ collisions is the nuclear-modification factor $R_{\mathrm{pPb}}$, calculated as

$$
R_{\mathrm{pPb}}=\frac{1}{A} \frac{\mathrm{d} \sigma_{\mathrm{pPb}} / \mathrm{d} p_{\mathrm{T}}}{\mathrm{d} \sigma_{\mathrm{pp}} / \mathrm{d} p_{\mathrm{T}}}
$$

which is the ratio of the production cross section in $\mathrm{p}-\mathrm{Pb}$ collisions to that in pp collisions scaled by the lead mass number A (equal to 208). Figure 7 shows that the $R_{\mathrm{pPb}}$ values of D mesons are 


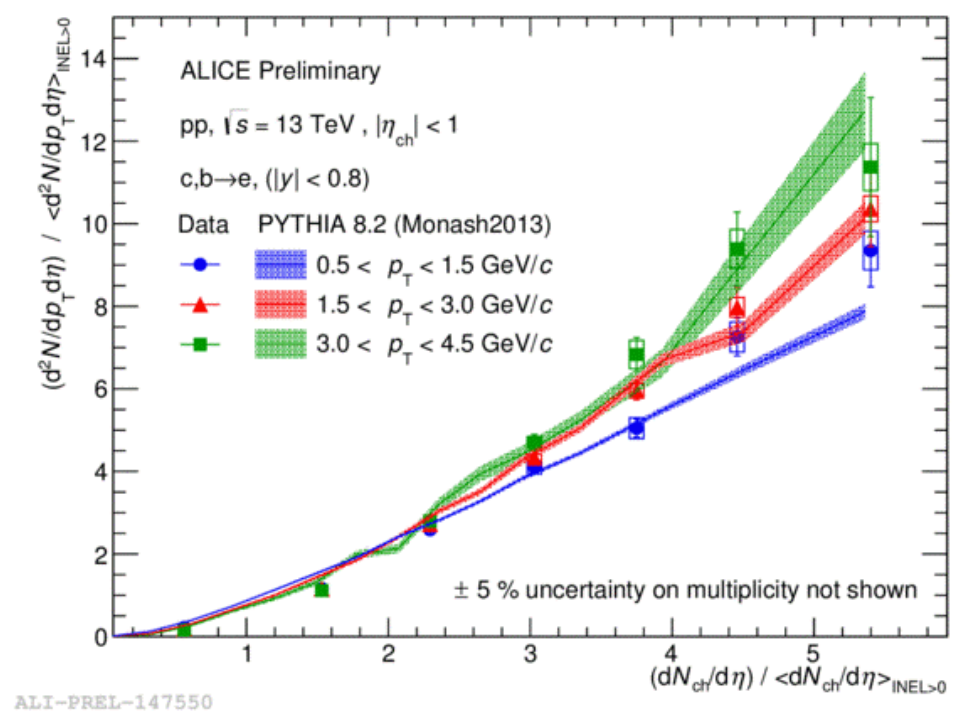

Figure 3: Heavy-flavour hadron decay electron self-normalised yields as a function of charged particle multiplicity compared to PYTHIA 8.2 results.

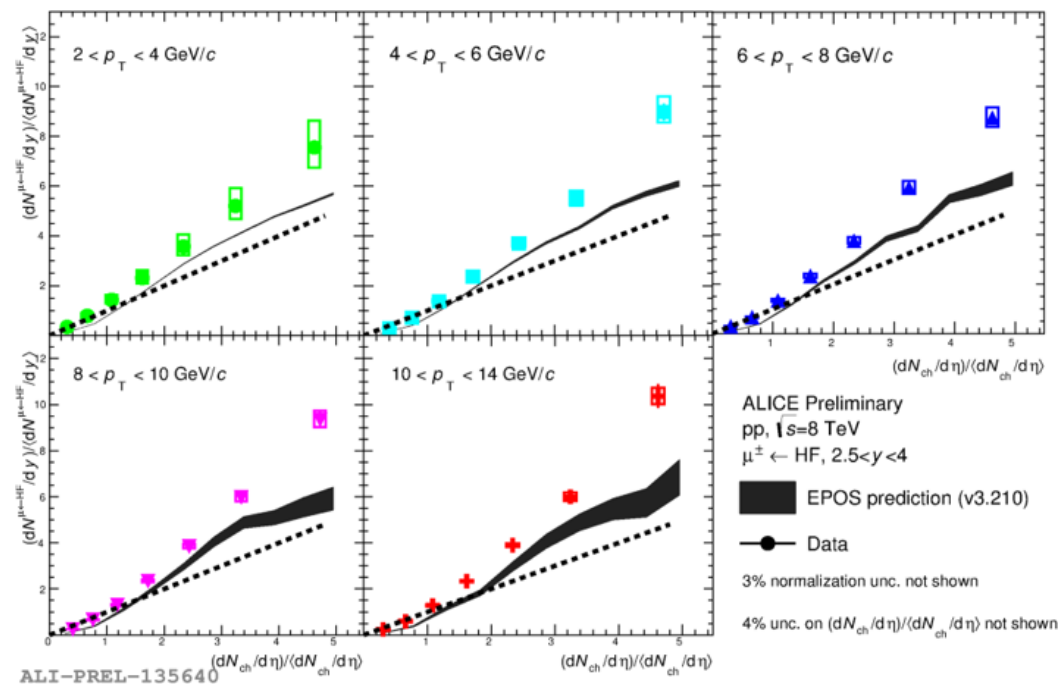

Figure 4: Heavy-flavour hadron decay muon self normalised yields as a function of charged particle multiplicity compared to EPOS predictions at forward rapidity.

compatible with unity in all the $p_{\mathrm{T}}$ intervals and the results are described by POWHEG+PYTHIA6 predictions. For the $\Lambda_{\mathrm{c}}^{+}$a significant suppression of the production below $2 \mathrm{GeV} / c$ is visible, while the $R_{\mathrm{pPb}}$ tends to be above unity elsewhere. This trend could indicate a possible radial flow effect or a modification of the hadronization mechanisms in $\mathrm{p}-\mathrm{Pb}$ collisions.

The data are compatible with POWHEG+PYTHIA6 up to $2 \mathrm{GeV} / c$ and with POWLANG up to $4 \mathrm{GeV} / c$, but in the intermediate and high $p_{\mathrm{T}}$ intervals both models are not able to describe the $\Lambda_{\mathrm{c}}^{+}$-baryon $R_{\mathrm{pPb}}$. 


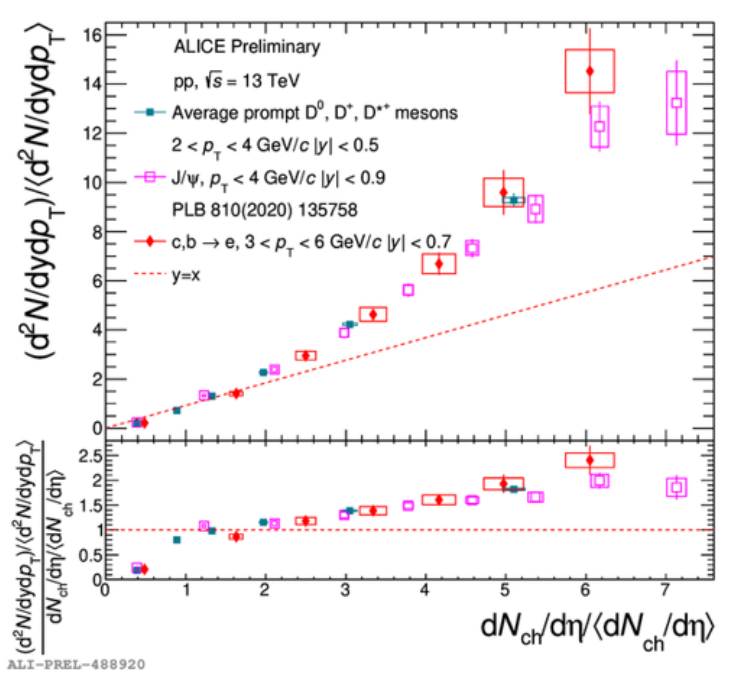

(a)

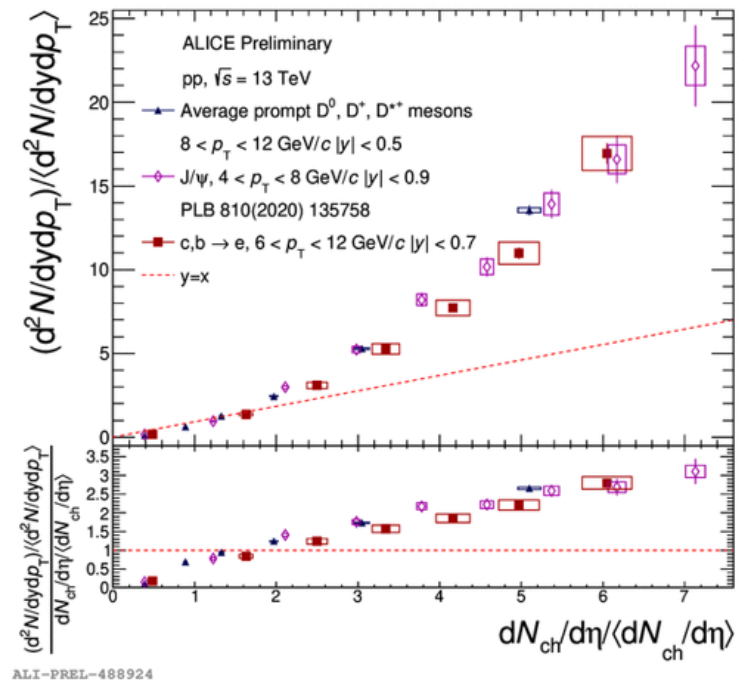

(b)

Figure 5: Comparison of self-normalised yields of D-meson average, $\mathrm{J} / \psi$ and heavy-flavour electrons at low (a) and high (b) $p_{\mathrm{T}}$ in pp collisions at $\sqrt{s}=13 \mathrm{TeV}$.

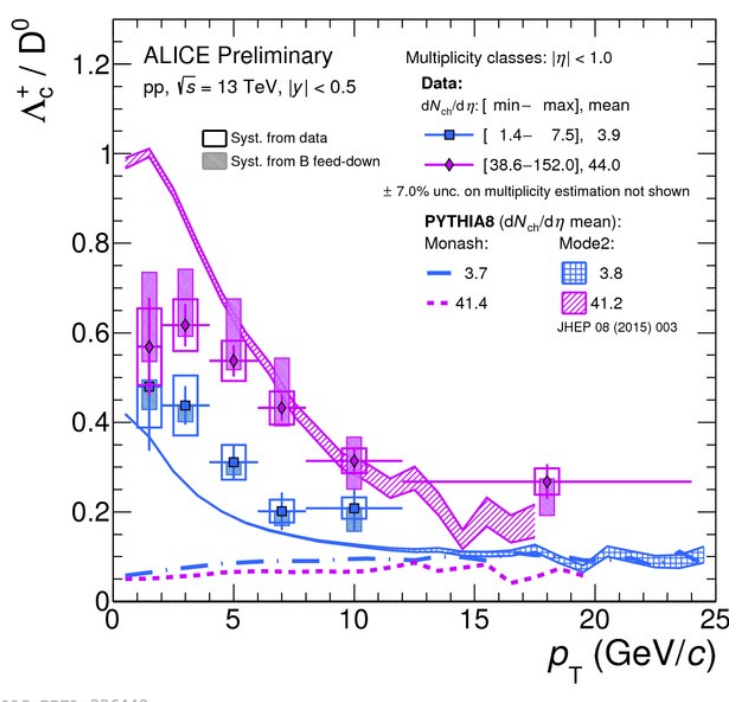

(a)

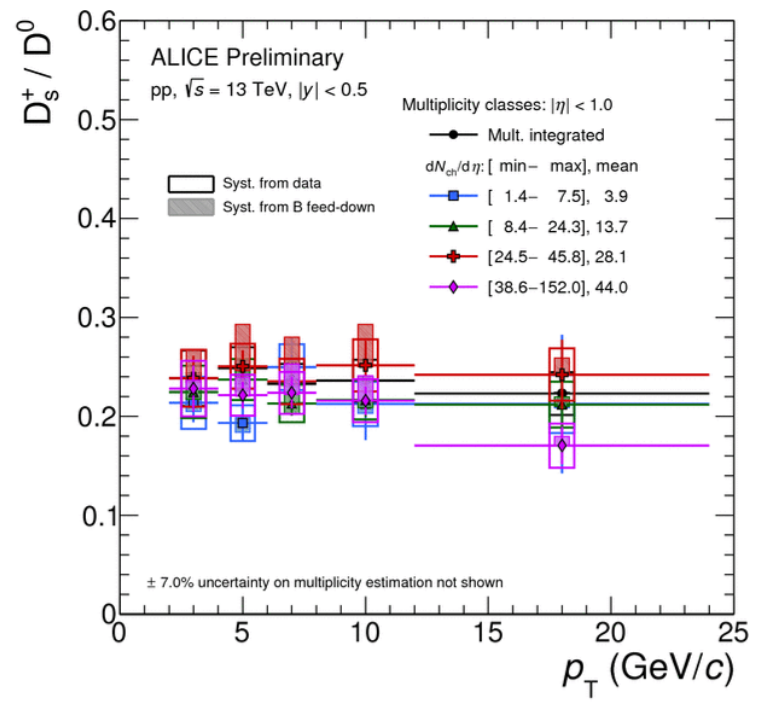

(b)

Figure 6: (a) $\Lambda_{\mathrm{c}}^{+} / \mathrm{D}^{0}$ and (b) $\mathrm{D}_{\mathrm{s}}^{+} / \mathrm{D}^{0}$ ratios in different multiplicity intervals as a function of $p_{\mathrm{T}}$. The $\Lambda_{\mathrm{c}}^{+} / \mathrm{D}^{0}$ data are compared to PYTHIA8 expectation.

The $Q_{\mathrm{CP}}$ of $\mathrm{D}$ mesons in $\mathrm{p}-\mathrm{Pb}$ collisions at $\sqrt{s_{\mathrm{NN}}}=5.02 \mathrm{TeV}$ was also measured. It is defined as the ratio of prompt D-meson yield in a defined multiplicity interval with that in the 60-100\% centrality and it is computed as

$$
Q_{\mathrm{CP}}=\frac{\left(\mathrm{d}^{2} N^{\text {prompt D }} / \mathrm{d} p_{\mathrm{T}} \mathrm{d} y\right)_{\mathrm{pPb}}^{\mathrm{i}} /\left\langle T_{\mathrm{pPb}}\right\rangle_{\mathrm{i}}}{\left(\mathrm{d}^{2} N \text { prompt D } / \mathrm{d} p_{\mathrm{T}} \mathrm{d} y\right)_{\mathrm{pPb}}^{60-100 \%} /\left\langle T_{\mathrm{pPb}}\right\rangle_{60-100 \%}}
$$




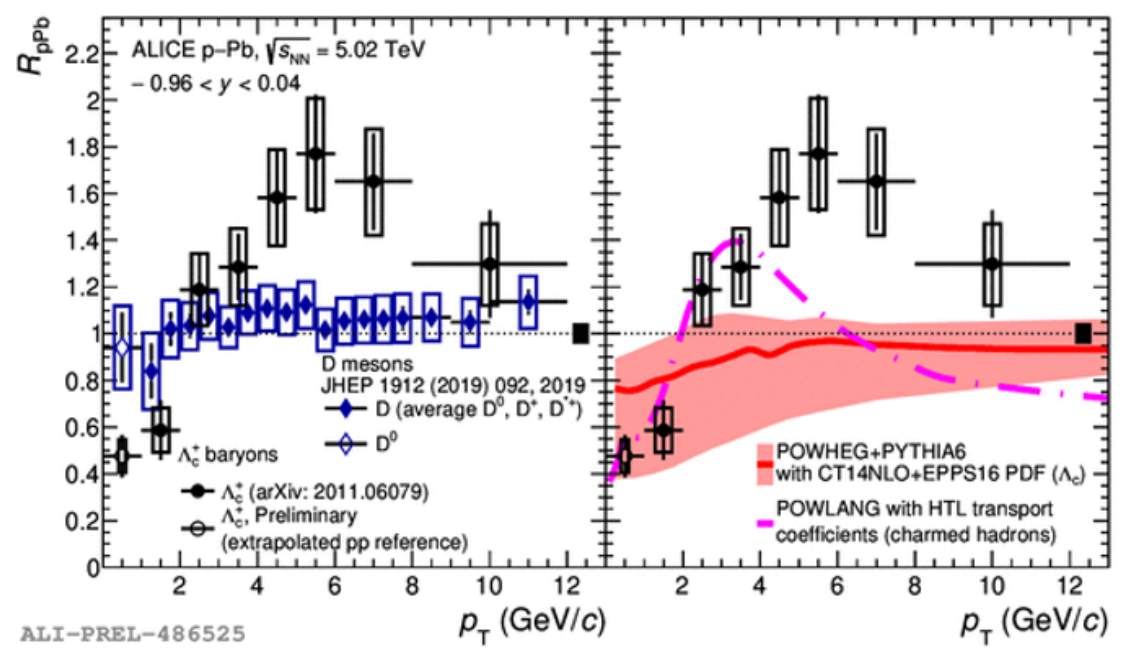

Figure 7: $\Lambda_{\mathrm{c}}^{+} R_{\mathrm{pPb}}$ as a function of $p_{\mathrm{T}}$ compared to $\mathrm{D}$ mesons results (left) and models (right) in $\mathrm{p}-\mathrm{Pb}$ collisions at $5.02 \mathrm{TeV}$.

where $\left\langle T_{\mathrm{pPb}}\right\rangle_{\mathrm{i}}$ is the nuclear overlap function in a defined $i$ multiplicity class.

In Fig. 8 the $Q_{\mathrm{CP}}$ is reported in four different centralities as a function of $p_{\mathrm{T}}$ and a hint of a $Q_{\mathrm{CP}}$ larger than unity at intermediate $p_{\mathrm{T}}$ is present, which might be caused by radial flow phenomena. In the figure also charged-particle data are shown and which are consistent with the D-meson ones [7].

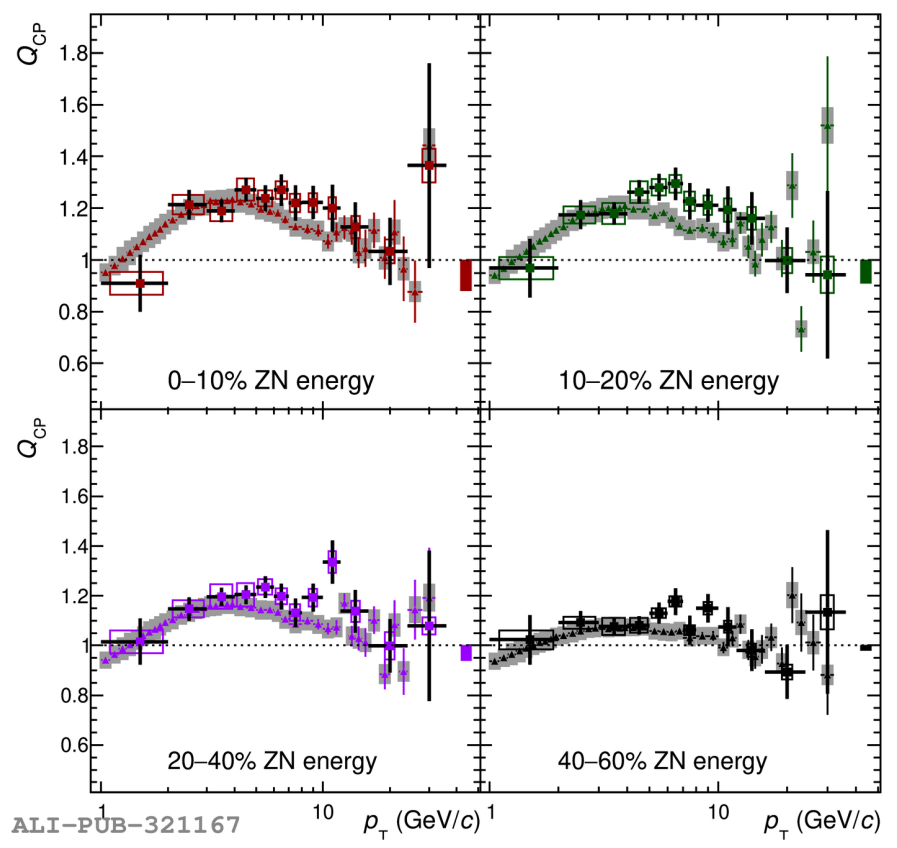

$$
\begin{aligned}
& \text { ALICE } \\
& \mathrm{p}-\mathrm{Pb}, \sqrt{S_{\mathrm{NN}}}=5.02 \mathrm{TeV} \\
& -0.96<y_{\mathrm{cms}}<0.04 \\
& \text { Prompt } \mathrm{D} \text { mesons } \\
& \square \text { Syst. on } \mathrm{d} N / \mathrm{d} p_{\mathrm{T}} \\
& \square \text { Syst. on }\left\langle T_{\mathrm{pPb}}\right\rangle \\
& \text { Charged particles } \\
& \text { Syst. on } \mathrm{d} N / \mathrm{d} p_{\mathrm{T}}
\end{aligned}
$$

Figure 8: D-mesons $Q_{\mathrm{CP}}$ in 4 multiplicity intervals as a function of $p_{\mathrm{T}}$ [7].

The elliptic flow $\left(v_{2}\right)$ was also measured for muons at forward rapidity and for heavy-flavour decay electrons at midrapidity in $\mathrm{p}-\mathrm{Pb}$ collisions at $\sqrt{s_{\mathrm{NN}}}=8.16$ and $5.02 \mathrm{TeV}$, respectively. The 
results, displayed in Fig. 9, are compatible between the two lepton species. Both of them show positive elliptic flow in more central events, which gives us an indication that collective phenomena might be present in $\mathrm{p}-\mathrm{Pb}$ collisions.

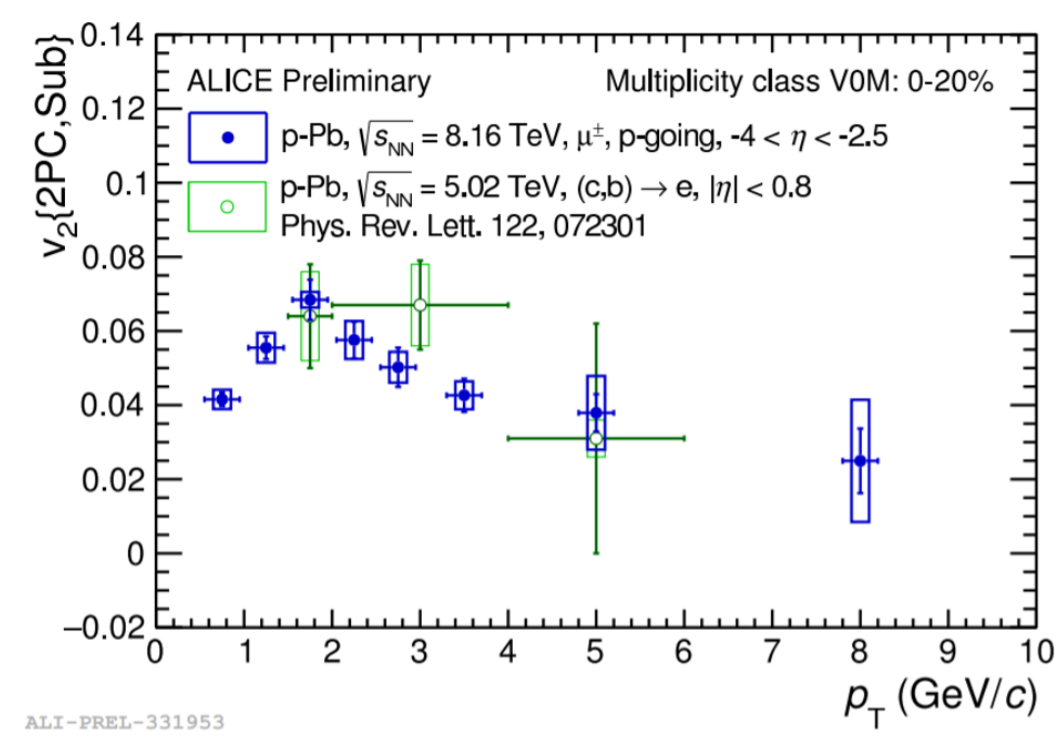

Figure 9: Elliptic flow of muons in $\mathrm{p}-\mathrm{Pb}$ collisions at $8.16 \mathrm{TeV}$ and heavy-flavour electrons at $\sqrt{s_{\mathrm{NN}}}=5.02$ $\mathrm{TeV}$ as a function of $p_{\mathrm{T}}$.

\section{Summary}

The self-normalised yield measurements in pp collisions at $\sqrt{s}=13 \mathrm{TeV}$ of $\mathrm{D}$ mesons, $\mathrm{J} / \psi$ and heavy-flavour decay electrons are compatible in similar $p_{\mathrm{T}}$ intervals: they all increase faster than linearly as a function of multiplicity and a significant $p_{\mathrm{T}}$ dependence was found. The results are reproduced well by EPOS3 with the hydrodinamic evolution for the D-meson average, while heavy-flavour electrons are described well by PYTHIA8 using the Monash 2013 tune.

Muon production at forward rapidity from heavy-flavour decays shows also a faster than linear trend vs. multiplicity.

The production ratio $\Lambda_{c}^{+} / \mathrm{D}^{0}$ shows an enhancement compared to $\mathrm{e}^{+} \mathrm{e}^{-}$results, while similar values are observed for D-meson species ratios. The data are well reproduced by PYTHIA8 CR mode 2 tune. The observed trend suggests that charm fragmentation might not be universal across different collision systems.

In $\mathrm{p}-\mathrm{Pb}$ collisions at $\sqrt{s_{\mathrm{NN}}}=5.02 \mathrm{TeV}$ the $\Lambda_{\mathrm{c}}^{+}$production was analysed. The $R_{\mathrm{pPb}}$ is compatible with POWLANG and POWHEG+PYTHIA6 only in lower $p_{\mathrm{T}}$ intervals.

The measurements of D-meson $Q_{\mathrm{CP}}$ and of the elliptic flow of muons and electrons from heavyflavour hadron decays suggest that collectivity phenomena could have a role in high-multiplicity $\mathrm{p}-\mathrm{Pb}$ collisions. 


\section{References}

[1] The ALICE Collaboration, Performance of the ALICE experiment at the CERN LHC, Int. J. Mod. Phys. A 29 (2014) 1430044

[2] Adam, J. et Al, Measurement of charm and beauty production at central rapidity versus charged-particle multiplicity in proton-proton collisions at $\sqrt{s}=7 \mathrm{TeV}$, Journal of High Energy Physics (2015) 148

[3] Werner, $\mathrm{K}$ et al, Analyzing radial flow features in $p-P b$ and $p$ - $p$ collisions at several TeV by studying identified-particle production with the event generator EPOS3, Phys. Rev. C 89, 064903 (2014)

[4] Schmidt, I. et al, Production mechanisms of open-heavy flavor mesons, Phys. Rev. D 101, $094020(2020)$

[5] Christiansen, J.R. and Skands, P.Z., String formation beyond leading colour, Journal of High Energy Physics 8 (2015) 3

[6] Gladilin, L., Fragmentation fractions of $c$ and $b$ quarks into charmed hadrons at LEP, Eur. Phys. J. C (2015) 75:19

[7] Acharya, S. et al for the ALICE Collaboration, Measurement of prompt $D^{0}, D^{+}, D^{*+}$, and $\mathrm{D}_{\mathrm{S}}^{+}$ production in $p-P b$ collisions at $\sqrt{\mathrm{s}_{\mathrm{NN}}}=5.02 \mathrm{TeV}$, Journal of High Energy Physics 12 (2019) 092 